\title{
ASYMPTOTIC NONUNIQUENESS OF THE NAVIER-STOKES EQUATIONS IN KINETIC THEORY ${ }^{1}$
}

\author{
BY RICHARD S. ELLIS AND MARK A. PINSKY \\ Communicated by H. Kesten, May 22, 1974
}

We consider the linearized Boltzmann equation

$$
\partial p / \partial t+\xi \cdot \operatorname{grad} p=Q p / \epsilon,
$$

whose solution $p=p_{\epsilon}(t, x, \xi), t>0, x \in R^{3}, \xi \in R^{3}, \epsilon>0 . Q$ is the linearized collision operator corresponding to a spherically symmetric hard potential, and $\epsilon$ is a parameter which represents the mean free path.

In a series of basic papers, Grad [6], [7], [8] studied the existence and asymptotic behavior of the solution of the initial value problem for (1), where the initial data $p_{\epsilon}\left(0^{+}, x, \xi\right)=f(x, \xi)$ satisfies mild growth and smoothness conditions. Grad's method begins with the decomposition

$$
Q=-\nu+K
$$

where $\nu$ is the operator of multiplication by the collision frequency $\nu(\xi)$, a strictly positive function of $|\xi|$, and $K$ is a compact operator on the Hilbert space $H_{0}$ of functions $f(\xi)$ which satisfy

$$
\langle f, f\rangle \equiv\left(\frac{1}{\sqrt{2 \pi}}\right)^{3} \int|f(\xi)|^{2} \exp \left(-|\xi|^{2} / 2\right) d \xi<\infty .
$$

Using (2), Grad wrote (1) as an integral equation and then derived a priori estimates for the solution in the Hilbert space

$$
H \equiv L^{2}\left(R^{6},(1 / \sqrt{2 \pi})^{3} \exp \left(-|\xi|^{2} / 2\right) d x d \xi\right) .
$$

Grad also related the asymptotic behavior of $p_{\epsilon}$ to the solutions of the linear Euler and Navier-Stokes equations. Given $f \in H$, define

AMS (MOS) subject classifications (1970). Primary 82A40, 76D30; Secondary 15A27, 76Q05, 45M05.

Key words and phrases. Boltzmann equation, hydrodynamic limit, Euler equations, Navier-Stokes equations, contraction semigroup.

1 Research supported by grant GP-28576. 


$$
\begin{aligned}
& f_{0}(x)=\langle f(x, \cdot), 1\rangle ; \\
& f_{i}(x)=\left\langle f(x, \cdot), \xi_{i}\right\rangle, \quad i=1,2,3 ; \\
& f_{4}(x)=\left\langle f(x, \cdot),\left(|\xi|^{2}-3\right) / \sqrt{ } 6\right\rangle,
\end{aligned}
$$

where $\langle\cdot, \cdot\rangle$ denotes the inner product on $H_{0}$. The Navier-Stokes equations are written

$$
\begin{aligned}
\partial n_{0} / \partial t+\operatorname{div} \mathbf{n} & =0 \\
\partial \mathbf{n} / \partial t+\operatorname{grad} n_{0}+\sqrt{2 / 3} \operatorname{grad} n_{4} & =\epsilon \eta[\Delta \mathbf{n}+(1 / 3) \operatorname{grad} \operatorname{div} \mathbf{n}], \\
\partial n_{4} / \partial t+\sqrt{2 / 3} \operatorname{div} \mathbf{n} & =\epsilon \lambda \Delta n_{4}, \\
n_{i}\left(0^{+}, \cdot\right) & =f_{i} .
\end{aligned}
$$

In (3), $\epsilon>0, n_{i}=n_{i}^{\epsilon}(t, x)(i=0, \cdots, 4), \mathbf{n}=\left(n_{1}, n_{2}, n_{3}\right)$, and $\eta>0$ and $\lambda>0$ are physical constants. The Euler equations are obtained from (3) by putting $\epsilon=0$. Setting

$$
\begin{aligned}
p_{\epsilon} & =T_{\epsilon}(t) f, \\
N_{\epsilon}(t) f & =n_{0}^{\epsilon}+\sum_{i=1}^{3} n_{i}^{\epsilon} \xi_{i}+n_{4}^{\epsilon} \frac{|\xi|^{2}-3}{\sqrt{ } 6}, \\
E(t) f & =N_{0}(t) f
\end{aligned}
$$

Grad proved the following asymptotic results:

$$
\begin{gathered}
T_{\epsilon}(t) f=E(t) f+O(\epsilon), \\
T_{\epsilon}(t / \epsilon) f=N_{\epsilon}(t / \epsilon) f+O(\epsilon) .
\end{gathered}
$$

In physical terms, (4) describes the nonviscous fluid approximation at a fixed time $t>0$; (5) describes the viscous effects when $t \rightarrow \infty$. Our aim is to show that (5) is only one of a large variety of possible refinements of (4). This is accomplished by the following two results.

Boltzmann Limit THEOREM. Let $f(x, \xi)$ be sufficiently regular. Then

$$
E(-t / \epsilon) T_{\epsilon}(t / \epsilon) f=\bar{N}(t) f+O(\epsilon) \quad(\epsilon \downarrow 0),
$$

where $\bar{N}(t)$ is a contraction semigroup on $H$ whose generator is given by the differential equations 


$$
\begin{aligned}
\frac{\partial n_{0}}{\partial t} & =\left(\frac{9}{25} \lambda+\frac{2}{5} \eta\right) \Delta n_{0}+\sqrt{\frac{2}{3}}\left(-\frac{6}{25} \lambda+\frac{2}{5} \eta\right) \Delta n_{4}, \\
\frac{\partial \mathbf{n}}{\partial t} & =\eta \Delta \mathbf{n}+\left(\frac{\lambda}{5}-\frac{\eta}{3}\right) \operatorname{grad} \operatorname{div} \mathbf{n}, \\
\frac{\partial n_{4}}{\partial t} & =\sqrt{\frac{2}{3}}\left(-\frac{6}{25} \lambda+\frac{2}{5} \eta\right) \Delta n_{0}+\left(\frac{11}{25} \lambda+\frac{4}{15} \eta\right) \Delta n_{4}, \\
n_{i}\left(0^{+}, x\right) & =f_{i}(x)
\end{aligned}
$$

i.e.,

$$
\bar{N}(t) f=n_{0}+\sum_{1}^{3} n_{i} \xi_{i}+n_{4} \frac{|\xi|^{2}-3}{\sqrt{ } 6} .
$$

The semigroup $\{\bar{N}(t), t \geqslant 0\}$ commutes with the Euler semigroup $\{E(t), t \geqslant 0\}$.

In order to make connection with (5) we also need the following.

NAvier-Stokes Limit TheOREm. Let $f(x, \xi)$ be sufficiently regular. Then

$$
E(-t / \epsilon) N_{\epsilon}(t / \epsilon) f=\bar{N}(t) f+O(\epsilon) \quad(\epsilon \downarrow 0) .
$$

The proof of (8) proceeds by means of Fourier transformation from the following purely algebraic result, of independent interest.

MAtrix Limit Theorem. Let $A, B$ be real, symmetric $m \times m m a$ trices and assume that $B$ is negative semidefinite. Then

$$
\exp (-i t A / \epsilon) \exp (t(i A+\epsilon B) / \epsilon)=\exp \left(t \pi_{A} B\right)+O(\epsilon) \quad(\epsilon \downarrow 0),
$$

where $\pi_{A} B$ is the orthogonal projection, in the space of $m \times m$ matrices, of $B$ onto the linear subspace of matrices which commute with $A$.

In particular, we show that $\bar{N}(t)$ is obtained by a projection, in the space of operators, of $N_{\epsilon}(t)$ upon the set of operators which commute with $\{E(t), t \geqslant 0\}$.

Using (6), we have

$$
T_{\epsilon}(t / \epsilon) f=E(t / \epsilon) \bar{N}(t) f+O(\epsilon) \quad(\epsilon \downarrow 0) .
$$

This is the simplest of an infinite number of alternatives to (5). Indeed, if $\widetilde{N}(t)$ is any operator whose projection is $\bar{N}(t)$, then we may substitute $\widetilde{N}(t)$ for $\bar{N}(t)$ in (9).

The proof of (6) depends on a careful spectral analysis of the operator $Q-i(\gamma \cdot \xi)$, where $\gamma \in R^{3}$ is a parameter. We prove the existence and 
differentiability, for $|\gamma|$ sufficiently small, of the hydrodynamical eigenvalues and eigenfunctions $\left\{\alpha^{(j)}(\gamma), e^{(j)}(\gamma) ; j=1, \cdots, 5\right\}$ which satisfy $\alpha^{(j)}(0)=0$, $e^{(j)}(0) \in \operatorname{span}\left\{1, \xi_{1}, \xi_{2}, \xi_{3},|\xi|^{2}\right\}$. We then prove a contour integral representation

$$
\begin{aligned}
\exp [t(Q-i(\gamma \cdot \xi))] f= & \sum_{j=1}^{5} \exp \left(t \alpha^{(j)}\right)(\gamma)\left\langle f, e^{(j)}(-\gamma)\right\rangle e^{(j)}(\gamma) \\
& +\frac{1}{2 \pi i} \int_{C} e^{t \alpha} R(\alpha, \gamma) \frac{(Q-i(\gamma \cdot \xi))^{2}}{\alpha^{2}} f d \alpha
\end{aligned}
$$

where $C$ is a vertical contour in the half plane $\operatorname{Re} \alpha<0$ and $R(\alpha, \gamma) \equiv$ $(Q-i(\gamma \cdot \xi)-\alpha)^{-1}$. The first term of (10) corresponds to the Hilbert solution and gives the connection with hydrodynamics. The second term is negligible in the hydrodynamic limit. In case $\nu(\xi) \sim|\xi|^{\alpha}$ as $|\xi| \rightarrow \infty \quad(\alpha>0)$, the contour integral may be replaced by $\int_{C} e^{t \alpha} R(\alpha, \gamma) f d \alpha$, where the contour $C$ is such that $\operatorname{Re} \alpha \rightarrow-\infty$ when $\operatorname{Im} \alpha \rightarrow \pm \infty$. The existence of the eigenvalues $\alpha^{(j)}(\gamma)$ follows by applying the implicit function theorem to the exact hydrodynamical dispersion laws. Previously, exact dispersion laws were obtained [11] only for hard sphere potentials, i.e., $\nu(\xi) \sim|\xi|$ as $|\xi| \rightarrow \infty$. In this case, the $\alpha^{(j)}(\gamma)$ are analytic functions and can also be obtained from Rellich's perturbation theorem [9], [10]. In case $\nu(\xi) \sim|\xi|^{\alpha}$ as $|\xi| \rightarrow \infty$, $0 \leqslant \alpha<1$, the $\alpha^{(j)}(\gamma)$ will not be analytic around $\gamma=0$. Nevertheless, we obtain an asymptotic development

$$
\alpha^{(j)}(\gamma) \sim \sum_{n=1}^{\infty} \alpha_{n}^{(j)}|\gamma|^{n} \quad(1 \leqslant j \leqslant 5)
$$

where $\alpha_{1}^{(j)}$ is imaginary and $\alpha_{2}^{(j)}<0$. These constants can be computed by formal perturbation theory. They correspond to the adiabatic sound speed and absorption coefficients for low frequency sound waves [5].

The results (6) and (8) extend known results on finite-state velocity models in one dimension [1], [2] to the full three-dimensional linearized Boltzmann equation. These theorems are valid in any number of dimensions. Their proofs and related matters will appear in full detail in [3], [4]

\section{REFERENCES}

1. R. Ellis and M. Pinsky, Limit theorems for model Boltzmann equations with several conserved quantities, Indiana Univ. Math. J. 23 (1973), 287-307. MR 47 \#058.

2. Asymptotic equivalence of the linear Navier-Stokes and heat equations in one dimension, J. Differential Equations (to appear).

3. R. Ellis and M. Pinsky, Projection of the Navier-Stokes equations upon the Euler 
equations, J. Math. Pures Appl. (to appear).

4. The first and second fluid approximations to the Boltzmann equation, J. Math. Pures Appl. (to appear).

5. J. D. Foch and G. W. Ford, The dispersion of sound in monoatomic cases, Studies in Statistical Mechanics, vol. 5, North-Holland, Amsterdam, 1970.

6. H. Grad, Asymptotic theory of the Boltzmann equation. II, Rarefied Gas Dynamics (Proc. Third Internat. Sympos., Palais de 1'UNESCO, Paris, 1962), vol. 1, Academic Press, New York, 1963, pp. 26-59. MR 27 \#6577.

7. Asymptotic equivalence of the Navier-Stokes and non-linear Boltzmann equations, Proc. Sympos. Appl. Math., vol. 17, Amer. Math. Soc., Providence, R. I., 1965, pp. 154-183. MR 32 \#1979.

8. Solution of the Boltzmann equation in an unbounded domain, Comm. Pure Appl. Math. 18 (1965), 345-354. MR 32 \#8913.

9. T. Kato, Perturbation theory for linear operators, Die Grundlehren der math. Wissenschaften, Band 132, Springer-Verlag, New York, 1966. MR 34 \#3324.

10. J. A. McLennan, Convergence of the Chapman-Enskog expansion for the linearized Boltzmann equation, Phys. Fluids 8 (1965), 1580-1584. MR 33 \#6948.

11. B. Nicolaenko, Dispersion laws for plane wave propagation, The Boltzmann Equation (ed. by F. A. Grünbaum), Courant Institute of Mathematical Sciences, New York, 1971, pp. 125-173.

DEPARTMENT OF MATHEMATICS, NORTHWESTERN UNIVERSITY, EVANSTON, ILLINOIS 60201 